\title{
Vorwort zur deutschen Übersetzung
}

Diese Einführung wendet sich zunächst an Studierende der Physik und der Natur- und Ingenieurwissenschaften an Universitäten und Fachhochschulen. Sie ist aber gleichermaßen als Lern- und Lehrbuch in der Weiterbildung und für das Selbststudium geeignet. Im englischsprachigen Raum und darüber hinaus ist dieses Werk wegen seines Inhalts, vor allem aber wegen seiner pädagogisch-didaktisch gelungenen Darstellung seit vielen Jahren sehr erfolgreich.

Unter Betonung der Prinzipien der klassischen und modernen Physik spannt es den Bogen von einfachen und grundlegenden bis zu praktischen Fragestellungen. Dabei wird die Einheit von theoretischer und Experimentalphysik ebenso deutlich wie die Rolle, die die Mathematik bei der Formulierung physikalischer Aussagen spielt.

Ein wesentlicher Vorteil dieses Buches und eine beträchtliche Erleichterung für die Lernenden sind die vielen, im Detail ausgeführten und gerechneten Beispiele sowie die äußerst umfangreiche Sammlung von überwiegend anwendungsbezogenen Aufgaben, die zur besseren Übersicht nach den einzelnen Abschnitten und in aufsteigendem Schwierigkeitsgrad geordnet sind.

Die vorliegende deutschsprachige Ausgabe basiert auf der 3. bzw. 4. amerikanischen Auflage. Nomenklatur und Einheiten wurden dem von der Internationalen Union für reine und angewandte Physik (IUPAP) empfohlenen Standard und weitgehend auch den DIN-Normen angepaßt.

Unser besonderer Dank gilt Herrn Dr.-Ing. Rudolf Weber für seine stete Unterstützung beim Zustandekommen dieses Buches. 
